\title{
EMBEDDING PHENOMENA BASED UPON DECOMPOSITION THEORY: WILD CANTOR SETS SATISFYING STRONG HOMOGENEITY PROPERTIES ${ }^{1}$
}

\author{
ROBERT J. DAVERMAN
}

\begin{abstract}
We point out the sharpness of earlier results of McMillan by exhibiting a map of the $n$-sphere $S^{n}, n>5$, onto itself having acyclic but non-cell-like polyhedra as its nondegenerate point inverses and for which the image of the set of nondegenerate point inverses is a Cantor set $K$. Of necessity, $K$ is wildly embedded, and it has the unusual additional property that every self-homeomorphism of $K$ extends to a self-homeomorphism of $S^{n}$.
\end{abstract}

1. Introduction. According to work of D. R. McMillan, if $f$ is a map of $S^{n}$ to itself such that the image of the set of nondegenerate point inverses is 0-dimensional, then each point inverse is strongly acyclic over the integers (see [M] for definitions) and, in particular, has the integral Čech cohomology of a point [M, Lemma 5]; moreover, for the case $n=3$, each point inverse is cellular [M, Corollary 3.5]. We show here that for $n \geqslant 5$ this stronger conclusion of cellularity fails in what is known to be the simplest possible case, in which the image of the nondegenerate elements forms a Cantor set.

This image Cantor set $K$ must be wildly embedded (otherwise, $K$ would be defined by cells in $S^{n}$, and the inverse image of the defining cells would also be cells, implying that each nondegenerate point inverse is cellular). As an elementary by-product of its construction, $K$ is seen to possess a symmetry previously undiscovered in wild Cantor sets, for it is strongly homogeneously embedded, meaning that each homeomorphism of $K$ onto itself can be extended to a homeomorphism of $S^{n}$ to itself. Displaying a weaker form of symmetry, the classical examples of Antoine [A] and Blankenship [BI] are homogeneously embedded in the sense that, for any two points $p, q$ in such examples $X$, there is a homeomorphism $H$ of $S^{n}$ to itself for which $H(X)=$ $X$ and $H(p)=q$.

Some profound recent developments concerning decompositions of manifolds support what may appear to be the innocuously easy constructions of this paper. The first of these is due to Cannon [C], who showed that for a

\footnotetext{
Received by the editors July 25, 1978 and, in revised form, September 14, 1978.

AMS (MOS) subject classifications (1970). Primary 57A15, 57A45, 54B15; Secondary 57A40, $55 \mathrm{F5} 5$.

Key words and phrases. Cell-like, acyclic, upper semicontinuous, decomposition, disjoint disks property, generalized manifold, wild Cantor set, strongly homogeneous embedding.

'Research supported in part by NSF Grant MCS 76-07274.
} 
cell-like decomposition $G$ of an $n$-manifold $M(n \geqslant 5)$ such that the image of the nondegenerate elements is contained in a closed $k$-dimensional set $Y$ $(2 k+1 \leqslant n), M / G$ is a manifold (homeomorphic to $M$ ) if and only if $M / G$ satisfies the following disjoint disks property: any two maps of the 2-cell $I^{2}$ into $M / G$ can be approximated arbitrarily closely by maps having disjoint images. This has been improved by Edwards [E], who obtained the same result with no restriction on the image of the nondegenerate elements beyond the requirement that $M / G$ itself be finite dimensional. The second development, concerning the resolution of singularities in nonmanifolds, is due to Bryant and Lacher [BL], who showed that if $Y$ is a generalized $n$-manifold of dimension $n \geqslant 5$ that is known to be an $n$-manifold except possibly at points of some 0-dimensional closed subset $S(Y)$, then $Y$ is the cell-like image of an $n$-manifold. This result also has been improved, by Cannon, Bryant and Lacher [CBL], who obtained the same conclusion in case the potential nonmanifold set $S(Y)$ is contained in a closed subset of dimension $k$, where $2 k+2 \leqslant n$. An explicit consequence of the above needed for applications here is the following theorem.

Theorem A (CANNon, Bryant AND LACher). Suppose $Y$ is a generalized $n$-manifold, $n \geqslant 5$, such that

(1) $Y$ contains a 0-dimensional closed set $S(Y)$ such that $Y-S(Y)$ is an n-manifold, and

(2) $Y$ satisfies the disjoint disks property.

Then $Y$ is an n-manifold.

As in [BL] and [CBL], a generalized $n$-manifold is understood to be an ENR (Euclidean neighborhood retract $=$ a retract of an open subset of some Euclidean space) such that, for each $y \in Y$,

$$
H_{*}(Y, Y-\{y\} ; Z) \approx H_{*}\left(E^{n}, E^{n}-\{0\} ; Z\right) .
$$

2. The basic construction. McMillan [M, p. 959] presents an example of an acyclic but non-cell-like map $f$ of $S^{n}(n \geqslant 4)$ to itself such that the image of the nondegenerate elements is an arc. To a great extent the example described below represents a 0 -dimensional version of his.

Throughout the remainder of this paper $n$ will represent a fixed integer greater than 4.

Let $M^{n-2}$ be a compact PL homology $(n-2)$-cell (an $(n-2)$-manifoldwith-boundary having trivial homology groups but nontrivial fundamental group) and let $X^{\prime}$ be a PL $(n-3)$-spine for $M^{n-2}$, that is, $X^{\prime} \subset$ Int $M^{n-2}$ and $M^{n-2}$ collapses to $X^{\prime}$. Let $N^{n-1}=M^{n-2} \times[-1,1]$, which then has $X=X^{\prime} \times\{0\}$ as a spine and for which, in particular, $N^{n-1}-X \approx\left(\partial N^{n-1}\right)$ $\times[0,1)$. Let $C$ be the standard "middle thirds" Cantor set in $I=[0,1]$. Consider the upper semicontinuous decomposition $G$ of $Q=N^{n-1} \times[-2,2]$ having $\{X \times\{c\} \mid c \in C\}$ as its collection of nondegenerate elements. Let $Q^{*}$ 
denote the decomposition space $Q / G$ and $\pi: Q \rightarrow Q^{*}$ the decomposition map.

MaIN Lemma. The decomposition space $Q^{*}=Q / G$ is a compact $n$ manifold-with boundary.

Proof. Clearly the image of $\partial Q$ in $Q^{*}$ is a collared $(n-1)$-manifold. The argument here will establish that the image $Y$ of Int $Q$ is an $n$-manifold.

The space $Y$ contains a Cantor set $K$ of possible singular points, $K$ corresponding to the image under $\pi$ of the nondegenerate elements of $G$, such that $Y-K$ is an $n$-manifold. Not only does this mean that $Y$ fulfills condition (1) of Theorem A, it also implies that $Y$ is $n$-dimensional [HW, p. 32].

Next we show that $Y$ is locally contractible. This is obvious for points of $Y-K$. Since each point of $K$ has arbitrarily small (closed) neighborhoods homeomorphic to $Q^{*}$, it suffices to prove that $Q^{*}$ is contractible. The construction guarantees that $Q^{*}$ deformation retracts to $\pi(X \times I)$, and thus the problem reduces further to proving that $\pi(X \times I)$ is contractible. To do that, we name two auxiliary sets of maps. The first is a set of retractions $r_{c}$, defined for $c \in C$, of $\pi(X \times I)$ to $\pi(X \times[c, 1])$ sending $\pi(X \times[0, c])$ to the point $\pi(X \times\{c\})$. Before we name the second, we note that for each component $(a, b)$ of $I-C, \pi(X \times[a, b])$ is topologically the suspension of the acyclic polyhedron $X$ and, therefore, is contractible (see [S, Exercise 8.D.3, p. 461]). Then the second auxiliary set is a family of contractions, where, for each component $(a, b)$ of $I-C, \psi_{t}$ is a contraction of $\pi(X \times$ $[a, b])$, parametrized by $t \in[a, b]$, such that $\psi_{a}$ is the identity, $\psi_{t}(\pi(X \times\{b\}))$ is identically $\pi(X \times\{b\})$, and $\psi_{b}$ is the constant map to $\pi(X \times\{b\})$. Now we define a contradiction $h_{t}(t \in I)$ of $\pi(X \times I)$ as

$$
h_{t}(\pi(x, s))= \begin{cases}\pi(x, s) & \text { if } s>t, \\ r_{t} \pi(x, s) & \text { if } t \in C, \\ \psi_{t} r_{a}(x, s) & * * *\end{cases}
$$

where the convention $(* * *)$ governing the final part of this rule is that $s<t$ and $t$ lies in the component $(a, b)$ of $I-C$.

It follows that the finite dimensional, locally contractible separable metric space $Y$ is an ANR [H, Theorem V.7.1] and, therefore, is an ENR [L, p. 718]. Moreover, because each $\pi^{-1}(y)$ is acyclic, the Vietoris-Begle mapping theorem [Br, Theorem V.6.1] and standard duality theory [S, Theorem 6.9.10] yield that

$$
\begin{aligned}
H_{n-k}(Y, Y-\{y\}) & \approx H_{n-k}\left(\text { Int } Q, \text { Int } Q-\pi^{-1}(y)\right) \\
& \approx \bar{H}^{k}\left(\pi^{-1}(y)\right) \\
& \approx H^{k}(\text { point }) \\
& \approx H_{n-k}\left(E^{n}, E^{n}-\{0\}\right) .
\end{aligned}
$$


As a result, $Y$ is a generalized $n$-manifold.

Finally, we turn to condition (2) of Theorem A-the disjoint disks property. We first show that, for any dense subset $D$ of $K$, each map $f$ of $I^{2}$ into $Y$ can be approximated by a map of $I^{2}$ into $D \cup(Y-K)$. To do this, choose a triangulation $T$ of $I^{2}$ with very small mesh. Approximate $f$ by a map $g$ such that $g\left(T^{(1)}\right) \subset Y-\pi(X \times[-2,2])$ (here $T^{(1)}$ denotes the 1-skeleton of $\left.T\right)$, which is possible, of course, because $\operatorname{dim} \pi(X \times[-2,2]) \leqslant n-2$. Require this approximation $g$ to be so close to $f$ that, for those 2-simplexes $\sigma$ of $T$ such that $f(\sigma)$ misses $K, g(\sigma)$ also misses $K$. In case $f(\sigma) \cap K \neq \varnothing$, modify $g \mid \sigma$ once more in the following manner: $g \mid \partial \sigma$ is homotopic to a small loop $L$ near the cone point $\pi(X \times\{d\})$ in the space $\pi\left(N^{n-1} \times\{d\}\right), d \in D$ (which space is topologically the cone on $\left.\partial N^{n-1}\right)$, by a homotopy moving points along the images of vertical arcs from $Q=N^{n-1} \times[-2,2]$ and ranging through a small subset of $Y-\pi(X \times[-2,2]$; the loop $L$ then is contractible in a small subset of $\pi\left(N^{n-1} \times\{d\}\right)$. Define $g \mid \sigma$ as such a contraction of $g \mid \partial \sigma$.

In order to establish the disjoint disks property, we choose disjoint, dense subsets $D_{1}$ and $D_{2}$ of $K$. By the preceding paragraph, given maps $f_{i}$ of $I^{2}$ into $Y(i=1,2)$, we can approximate them by maps $g_{i}$ such that $g_{i}\left(I^{2}\right) \subset D_{i} \cup(Y$ $-K)(i=1,2)$. This means that $g_{1}\left(I^{2}\right)$ and $g_{2}\left(I^{2}\right)$ intersect only at points of the $n$-manifold $Y-K$. Consequently, we can exploit traditional general position methods to further adjust the maps $g_{i}$, changing things only at points of $g_{i}^{-1}(Y-K)$, to maps $h_{i}(i=1,2)$ such that

$$
h_{1}\left(I^{2}\right) \cap h_{2}\left(I^{2}\right)=\varnothing .
$$

As a consequence of Theorem A, $Q^{*}$ is an $n$-manifold-with boundary.

\section{The map of $S^{n}$ to itself.}

Proposition 1. There is a non-cell-like map $f$ of $S^{n}$ to itself $(n \geqslant 5)$ such that the image of the nondegenerate point inverses under $f$ is a Cantor set $K$.

Proof. Crucial to this argument is a fact established in the course of the main lemma that $Q^{*}$ is contractible.

Form a space $S$ from the disjoint union of $Q$ and $Q^{*}$ by identifying each point $x \in \partial Q$ with $\pi(x) \in Q^{*}$, form another space $T$ by doubling $Q^{*}$ along $\partial Q^{*}$ ( $T$ results from the disjoint union of two copies of $Q^{*}$ by identifying corresponding boundary points), and name a map $f$ of $S$ onto $T$ such that $f \mid Q$ acts like $\pi$ in taking $Q$ onto one of the copies of $Q^{*}$ and that $f \mid Q^{*}$ acts as the identity mapping onto the other copy of $Q^{*}$ in $T$. Then the set of nondegenerate point inverses of $f$ coincides with that of $\pi$, and its image under $f$ is a Cantor set $K$ in $T$.

Each of $S$ and $T$ is a closed $n$-manifold. By a simple Mayer-Vietoris calculation, each has the homology of $S^{n}$. Moreover, each is simply connected: since $Q^{*}$ is contractible, $\pi_{1}(S)$ is generated by the image of $\pi_{1}(Q)$, which in turn is generated by the image of $\pi_{1}(\partial Q)$, and which itself is contained in 
the (trivial) image of $\pi_{1}\left(Q^{*}\right)$; it is even more obvious that $T$ is simply connected. Hence, $S$ and $T$ are each topologically equivalent to $S^{n}[\mathbf{N}]$.

\section{Properties of the Cantor set.}

Proposition 2. There exists a wildly embedded, strongly homogenously embedded Cantor set $K$ in $S^{n}(n \geqslant 5)$. Furthermore, for each $\varepsilon>0$ there exists $\delta>0$ such that every homeomorphism $h$ of $K$ to itself moving points less than $\delta$ extends to a homeomorphism $H$ of $S^{n}$ to itself moving points less than $\varepsilon$ and fixed outside the $\varepsilon$-neighborhood of $K$.

Proof. The Cantor set $K$, of course, is the one determined in $\S 3$, where $K \subset Q^{*} \subset T \approx S^{n}$. As an alternative to the decomposition theory argument sketched in the introduction that $K$ is wild, consider a map $g: \partial I^{2} \rightarrow N^{n-1} \times$ $\{2\}$ defining a loop in $\partial Q$ that is not contractible in $Q$. Since $Q^{*}$ is contractible, $\pi g$ extends to a map $g^{*}$ of $I^{2}$ in $Q^{*}$. If $K$ were tame, $g^{*}$ could be adjusted, without changing the map on $\partial I^{2}$, to a map $g^{\prime}$ into $Q^{*}-K$. This leads to the contradiction that $\pi^{-1} g^{\prime}$ is a contraction of $g$ in $Q$.

As an aid for studying the embedding of $K$ in $Q^{*}$, we reconsider the source $Q$ as $M^{n-2} \times B$, with $B$ representing the 2-cell $[-1,1] \times[-2,2]$, and with $C=\{0\} \times C$ the Cantor set in Int $B$ for which $\pi\left(X^{\prime} \times C\right)=K$. The tameness of Cantor sets in the plane implies that each homeomorphism $h^{*}$ of $C$ onto itself extends to a homeomorphism $H^{*}$ of $B$ onto itself fixed on $\partial B$. Then, given any homeomorphism $h$ of $K$ to itself, one induces a homeomorphism $h^{*}=\pi^{-1} h \pi$ on $C$, extends $h^{*}$ to the promised homeomorphism $H^{*}$ on $B$, defines a homeomorphism $H$ on $Q^{*}=\pi\left(M^{n-2} \times\right.$ $B)$ as $\pi\left(I d \times H^{*}\right) \pi^{-1}$, and finally extends $H$ to other points of $T \approx S^{n}$ via the identity. Furthermore, because $C \subset B^{2}$ satisfies the stronger homogeneity property mentioned in the statement of the proposition, the argument just given shows that $K \subset S^{n}$ satisfies it as well.

\section{REFERENCES}

[A] L. Antoine, Sur l'homeomophie de deux figures et de leur voisinages, J. Math. Pures Appl. 86 (1921), 221-325.

[B1] W. A. Blankinship, Generalization of a construction of Antoine, Ann. of Math. (2) 53 (1951), 276-297.

[Br] G. E. Bredon, Sheaf theory, McGraw-Hill, New York, 1967.

[BL] J. L. Bryant and R. C. Lacher, Resolving zero-dimensional singularities in generalized manifolds, Math. Proc. Cambridge Philos. Soc. 83 (1978), 403-413.

[C] J. W. Cannon, Shrinking cell-like decompositions of manifolds. Codimension three (to appear).

[CBL] J. W. Cannon, J. L. Bryant and R. C. Lacher, The structure of generalized manifolds having nonmanifold set of trivial dimension (to appear).

[E] R. D. Edwards, Approximating certain cell-like maps by homeomorphisms (to appear). 
[H] S. T. Hu, Theory of retracts, Wayne State Univ. Press, Detroit, 1965.

[HW] W. Hurewicz and H. Wallman, Dimension theory, Princeton Univ. Press, Princeton, N. J., 1948.

[L] R. C. Lacher, Cell-like mappings. I, Pacific J. Math. 30 (1969), 717-731.

[M] D. R. McMillan, Jr., Acyclicity in three-manifolds, Bull. Amer. Math. Soc. 76 (1970), 942-964.

[N] M. H. A. Newman, The engulfing theorem for topological manifolds, Ann. of Math. (2) 84 (1966), 555-571.

[S] E. H. Spanier, Algebraic topology, McGraw-Hill, New York, 1966.

Department of Mathematics, University of Tennessee, KnoXVille, Tennessee 37916 